\title{
Lore Reich Rubin: Erinnerungen an eine chaotische Welt. Mein Leben als Tochter von Annie Reich und Wilhelm Reich
}

\author{
Psychosozial Verlag, 2019, Gießen, 253 Seiten, 29,90€(D)
}

$\mathrm{N}$ ach 65 Jahren kehrt Lore Reich Rubin im Jahr 2000 erstmals nach Wien zurück, die Stadt, in der sie geboren wurde und die sie seit ihrer Kindheit nicht mehr gesehen hatte. Sie genießt es, freundlich empfangen zu werden. Damit beginnen die Erinnerungen der jüngeren Tochter von Annie und Wilhelm Reich, die ein ganz anderes Bild von ihrem Vater entstehen lassen als die Bilder, die ihre ältere Schwester Eva, die später ausschließlich bewundernd von Wilhelm Reich sprach, und ihr Halbbruder Peter entworfen haben. Für sie war Reich nicht ein „Traumvater“, wie Peter seine Erinnerungen an den Vater betitelte, sondern eher ein Traumavater. Als Kind hatte Lore nämlich nicht viel Freundlichkeit erlebt. Es ist beklemmend, in dieser Autobiografie zu lesen, wie bindungslos sie aufwuchs. Ein Schicksal, das sie wohl mit vielen Kindern ihrer Zeit teilte.

1928 kommt Lore Reich Rubin zur Welt. 1929 reisen die Eltern für vier Monate in die Sowjetunion. Sie bleibt beim Kindermädchen zurück, zu dem sie die stärkste Bindung hatte. Als sie zwei Jahre alt ist, verlässt der Vater die Familie und geht nach Berlin. Dort hat er eine Freundin, lässt aber Frau und Kinder ein Jahr später nachkommen. Zu Hause tobt zwischen den Eltern Krieg. Lores Erinnerungen werden beherrscht von den Wutanfällen des Vaters und seiner Brüllerei. Oder davon, dass die Kinder sich selbst überlassen waren und still zu sein hatten, weil die Eltern in der Wohnung ihre Patienten empfingen. Gemeinsame Mahlzeiten gab es zu Hause fast nie. Der Vater sei in Cafés und Restaurants essen gegangen, während es für die Kinder zu Hause nur wenig gegeben habe. Er habe die Ansicht vertreten, Mutter und Vater sollten gleichmäßig in die Haus- haltskasse einzahlen, und was übrig bliebe, gehöre jedem von ihnen alleine. Nur war das bei ihm weit mehr als bei seiner Frau Annie.

Als Lore drei ist, werden sie und ihre Schwester Eva in Berlin für ein halbes Jahr in eine kommunistische Kinderkommune gegeben. Die Eltern kommen ein paar Male auf Besuch. Mit dem Ausbruch des Nationalsozialismus müssen die beiden alleine zu ihren Großeltern nach Wien fahren, die sie bald kommentarlos für drei Jahre einer fremden Frau übergeben, bei der sie in einer Art Kinderpension leben. Dort quartierten Eltern ihre Kinder ein, die bei Wiener Kinderpsychoanalytikern in Behandlung gingen. Auch Lores Schwester Eva lag regelmäßig auf der Couch, und daher musste die kleine Lore mit ihr da wohnen. Aber kein Kind durfte darüber sprechen, wie es ihm mit der Herzenskälte in der Kinderpension ging. Auch nicht, wenn einmal in der Woche die Eltern anriefen. 1934 ist der Vater in Dänemark, die Mutter bei ihrem Lehranalytiker in Prag, und die Kinder sind in Wien, damit Eva weiter zur Kinderanalyse gehen kann, und zwar bei einer Freundin der Mutter, Berta Bornstein, die dem Vater schreibt, er solle sich mit seiner kleinen Tochter nicht in Verbindung setzen, weil das die Analyse störe. Die Bindung zu schädigen war Teil des damaligen psychoanalytischen Behandlungskonzepts. Reich unterwarf sich diesem Diktat der Kontaktlosigkeit, das Anna Freud Evas Analytikerin auferlegt hatte. Auch versuchte die Analytikerin, der Tochter klarzumachen, dass ihr Vater verrückt sei und sie sich von ihm trennen solle. Es gab offensicht- 
lich keine Schamgrenze für die Diffamierung Reichs durch das psychoanalytische Establishment, selbst nicht gegenüber seiner Tochter. Als Lore 1936 zu ihrer Mutter nach Prag zieht, bleibt die zwölfjährige Schwester Eva nur aus dem Grund in Wien, dass sie ihre Kinderanalyse fortsetzen kann. Selbstverständlich im Liegen mit der Analytikerin hinter der Couch.

Das alles stellt Lore Reich Rubin in dieser interessanten Autobiografie dar. Wenn man das Buch liest, erschüttert einen die Grausamkeit, zu der die frühe Psychoanalyse fähig war. Aber auch die Herzlosigkeit von Annie und Wilhelm Reich kann man sich heute nur schwer vorstellen. Sicher war diese Teil einer Zeit, in der Kinder als emotionale Wesen nicht so geachtet wurden wie heute. Hinzu kam aber die Ideologie, die der Vater vertrat: „Mein Vater war der Meinung, dass Familie eine Institution der Bourgeoisie sei und dass man nicht nur mit jemandem verbunden sein sollte, weil dieser zur Familie gehörte. Das schloss natürlich auch Kinder ein, die nicht an ihre Eltern gebunden sein sollten." Der Vater wird von Lore Reich Rubin als ein vor allem auf sich selbst bezogener Mann beschrieben. Wenn ich daran zurückdenke, wie ich als junger Student mit Begeisterung die Massenpsychologie des Faschismus von Reich und seine Kritik an der autoritären Familie gelesen habe, wird mir ganz schwummerig. Dass Reich auch ein Vertreter der Bindungslosigkeit war, habe ich damals nicht gesehen. Und wer ihn heute noch als Vorläufer einer bindungsorientierten Kindertherapie sehen möchte, der sollte dieses Buch lesen. In Familie Reich zumindest schien alles andere Vorrang gehabt zu haben, nur nicht die Kinder. Lore wurde so zu einem tiefen Misstrauen erzogen, weil sie niemandem trauen konnte. Über ihre Mutter schreibt sie, in deren Leben sei sie „unwichtig“ gewesen.

Nur bei Kindermädchen oder Lehrern, wie in der Montessorischule in Wien, fand sie Verständnis. Kaum jemand half den Schwestern, zueinander zu finden. Sie wurden ihrer Ein- samkeit, ihrer Ratlosigkeit und ihrer verzweifelten Wut überlassen.

Auch in anderer Hinsicht wirft dieses Buch ein Licht auf Reich und seine Theorien. Als Lore mit sechs Jahren mit ihrem Vater zum psychoanalytischen Kongress in Luzern fuhr, schlief der Vater im Zelt, und sie musste voller Angst alleine im Auto schlafen. Angst durfte bei Reich anscheinend nicht sein. Dafür Wut und Aggression umso mehr: „Immer schrie und brüllte mein Vater“, er „schimpfte und tobte“. Wenn bis heute in manchen neoreichianischen Therapien aggressive Gefühlsäußerungen höher geschätzt werden als alles andere, so lebt darin vielleicht noch ein problematischer Charakterzug von Reich fort, unter dem seine Familie zu leiden hatte.

Auch Lore Reich Rubin scheint davon nicht frei zu sein. Offen gibt sie in ihrem Buch der tiefen Abneigung, ja ihrem Hass gegenüber ihrer Schwester Ausdruck, als müsste sie sich ihn immer noch von der Seele schreiben, während sie gleichzeitig meint, sich im Alter mit der 2008 verstorbenen Schwester versöhnt zu haben.

Ich stelle hier heraus, was wir aus diesem Buch über die frühe Psychoanalyse und über Reich erfahren, weil das für PsychotherapeutInnen interessant ist. Lore Reich Rubin rückt selbst das Verhältnis zu ihren Eltern auch ins Zentrum, aber ihr Buch geht darüber hinaus. Wir erfahren, was es für ein Kind heißen konnte, in jener Zeit in Europa zu leben. Als Tochter jüdischer Eltern wurde sie zwischen 1928 und 1938 auch deswegen hin- und hergeschoben, weil sie 1933 aus Deutschland fliehen mussten und Reich 1934 auch aus Dänemark, wo Lore mit ihm und seiner, wie sie es schildert, lebenslustigen Freundin Elsa Lindenberg vorher noch schöne Sommerferien am Strand verbracht hatte. Die Rückkehr nach Wien und dann der Umzug nach Prag sind für die Zehnjährige auch Stationen der Flucht.

1938 emigriert Annie Reich mit den Töchtern über Frankreich in die USA. Sie hat es ge- 
schafft, Visa zu besorgen. In New York beginnt sie, sich zusammen mit anderen Emigranten eine Existenz als Psychoanalytikerin aufzubauen. Für viele Jahre leben sie dort zusammen in einer kleinen Wohnung. Und das ist ein Weiteres, was wir aus diesem Buch erfahren: das Leben in der Emigration, die Eingliederung in eine fremde Kultur mit einer fremden Sprache und schließlich die Situation der politischen Linken in den USA nach dem Zweiten Weltkrieg, wo Lore und ihr Freund sich einer kleinen trotzkistischen politischen Partei anschließen und in dieser eine Zeitlang ihre Heimat finden, mit vorübergehender Aufgabe ihres Lebens als Intellektuelle.

1939 kommt auch der Vater aus Norwegen nach New York. Nach Jahren sieht Lore ihn wieder, einen Mann, mit dem sie Spaß haben konnte, der aber auch „unberechenbar und zornig“ war und der von der Tochter alles über ihre Sexualität wissen wollte, als sie eine keusche Teenagerin war. Der aber genauso wenig für sie da war wie die von ihrer Arbeit absorbierte Mutter.

Lore war gefangen zwischen der Welt einer Mutter, die Konfrontation und Gefühlsausbrüche vermied, wie sie schreibt, und einem autoritären Vater, der sie zur Unterwürfigkeit und Selbstentblößung gedrängt habe. Und der sie im Alter von 14 anbrüllte, sie solle aus seinem Leben verschwinden, als sie auf seine Frage, ob sie ihn für verrückt halte, mit Ja antwortete. Als sie ihn danach noch einmal in New York besucht, ignoriert er sie und redet nicht mit ihr. Nach Jahren sucht sie ihn später in Maine wie- der auf, wo er aber auch schon bald von ihr nichts mehr wissen will.

Im Unterschied zu dem Reich, den die Tochter Eva und der Sohn Peter schildern, ist der Reich, den Lore schildert, ein Vater, der die Schwestern spaltete und die eher aggressive Eva mehr zu mögen schien als die eher unglückliche Lore. Später fand Lore Briefe, in denen ihr Vater bei ihr 1934 - da war sie sechs Jahre alt - einen masochistischen Charakter diagnostizierte. Der Reich dieser Autobiografie ist ein hochgradig narzisstischer Mann, der sich in den USA „auf immer grandioser werdende Ziele konzentrierte“, bei denen er „die Grenzen seiner Ausbildung und seines Wissens" überschritten habe - was Lore als Kompensation seiner Verfolgung und seiner Angst, in Vergessenheit zu geraten, interpretiert, die er sich wohl nie zugestanden habe.

Lore fuhr zu seinem Begräbnis. Sie spricht vom „Begräbnis eines Kultführers“ mit verherrlichenden Reden. Nach Reichs Tod aber hätten die überlebenden Familienmitglieder zueinander gefunden, die er gespalten hatte, auch Lore und Reichs letzte Frau Ilse Ollendorf und deren Sohn Peter.

Das Buch zeigt eine in weiten Teilen tragische Geschichte einer Familie, in der ein narzisstischer Vater Bindung und Stabilität verhinderte, die Lore späterin einerlange anhaltenden Freundschaft und Ehe mit ihrem Mann Julie gefunden zu haben scheint.

Prof. Dr. Ulfried Geuter

DOI 10.2378/ktb2020.arto7d 